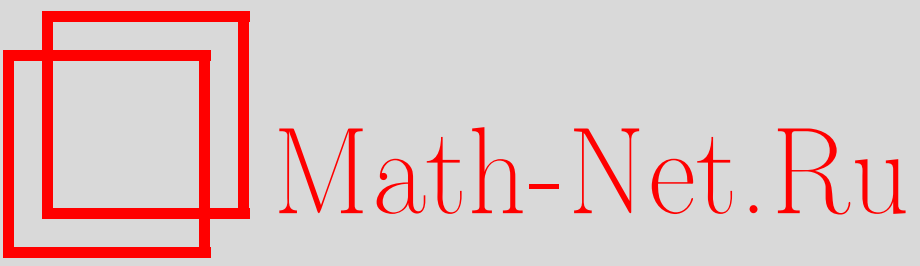

А. Н. Дранишников, О теории продолжения отображений компактов, УМН, 1998, том 53, выпуск 5, 65-72

DOI: https://doi.org/10.4213/rm70

Использование Общероссийского математического портала Math-Net.Ru подразумевает, что вы прочитали и согласны с пользовательским соглашением

http://www.mathnet.ru/rus/agreement

Параметры загрузки:

IP : 3.85 .183 .62

26 апреля 2023 г., 18:09:10 


\title{
О ТЕОРИИ ПРОДОЛЖЕНИЯ ОТОБРАЖЕНИЙ КОМПАКТОВ
}

\author{
А. Н. ДрАнишников
}

СОДЕРЖАНИЕ

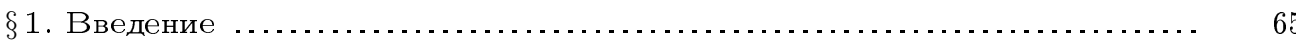

$\S 2$. Теорема Рубина-Шапиро и двойственная к ней $\ldots \ldots \ldots \ldots \ldots \ldots \ldots \ldots . .68$

$\S 3$. Теорема о букете ........................................ 70

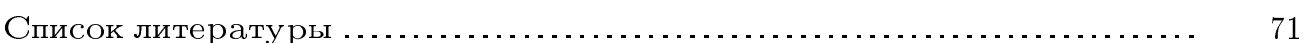

\section{$\S$ 1. Введение}

Задача продолжения на топологическом пространстве $X$ по отношению к пространству $K$ - это диаграмма

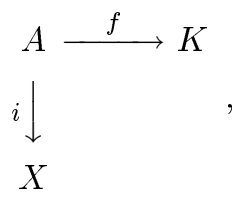

где $i: A \rightarrow X$ - замкнутое вложение, $f$-непрерывное отображение. Решением этой задачи продолжения служит непрерьвное продолжение $\bar{f}: X \rightarrow K$ отображения $f$ : $\bar{f} \circ i=f$. В этой статье мы рассматриваем только задачи продолжения в локально "хорошие" пространства $K$, такие как $C W$-комплексы или $A N E$-пространства. Мы напомним, что пространство $K$ назьвается абсолютным әкстензором для класса пространств $\mathscr{C}$, если все задачи продолжения на $X$ по отношению к $K$ для всех $X \in \mathscr{C}$ имеют решения. Обозначаем это как $K \in A E(\mathscr{C})$. Если мы интересуемся только продолжениями на некоторую открытую окрестность, мы получаем понятие $A N E(\mathscr{C})-$ абсолютного окрестностного әкстензора для класса $\mathscr{C}$. Теорема Борсука о продолжении гомотопии утверждает, что разрешимость задачи продолжения $f: A \rightarrow K$ в $C W$-комплекс $K$ для любого (нормального) пространства $X$ зависит только от гомотопического класса отображения $f$.

В случае, когда $K$ является простым комплексом, а $X$ - метрическим компактом размерности $\operatorname{dim} X<\infty$, разрешимость задачи продолжения может быть определена на языке теории препятствий [1]. В общем случае картина далека от ясности.

Работа выполнена при частичной поддержке NSF (грант № DMS-9696238). 
В двадщатые годы П. С. Александров (см. [2]) охарактеризовал лебегову размерность компактов в терминах продолжения отображений в сферы:

$\operatorname{dim} X \leqslant n \Longleftrightarrow$ все задачи продолжения на $X$ отображений в $n$-сферу $S^{n}$ разрешимы.

Когомологическая размерность может быть определена подобным образом:

$\operatorname{dim}_{G} X \leqslant n \Longleftrightarrow$ все задачи продолэения на $X$ отображений в комплекс Эйленберга-Маклейна $K(G, n)$ разрешимьл.

По аналогии, для любого пространства $W$ мы обозначаем через е- $\operatorname{dim} X \leqslant W$ свойство

(*) все задачи продолжения на $X$ отображений в $W$ разрешимы.

Это свойство обозначалось Куратовским как $X \tau W$. Другое обозначение для этого свойства - $W \in A E(X)$ вполне осмысленно: "W является абсолютньм ретрактом для класса, состояшего ровно из одного пространства $X$ ”. Здесь мы предпочитаем обозначение е- $\operatorname{dim} X \leqslant W$, так как оно также осмысленно и делает акцент на $X$. Итак, $\operatorname{dim} X \leqslant n \Leftrightarrow \mathrm{e}-\operatorname{dim} X \leqslant S^{n}$ и $\operatorname{dim}_{G} X \leqslant n \Leftrightarrow \mathrm{e}-\operatorname{dim}_{G} X \leqslant K(G, n)$.

Необходимость в глубоком изучении свойства е- $\operatorname{dim} X \leqslant W$ естественно возникает для метрического компакта $X$ и открытого многообразия $W$ в проблеме пересечения отображений [3], [4]. Теория была успешно развита до того места, которое позволило решить эту проблему во всех случаях, за исключением одного [5], [6]. Она получила имя “Теория Продолжения". Остающийся случай требует теории продолжения для неодносвязного $W$. Главное препятствие здесь следующее.

Проблема 1. Вытекает ли из условия $\operatorname{dim}_{\mathbb{Z}_{2}} X \leqslant 1$ условие $\mathrm{e}-\operatorname{dim} X \leqslant \mathbb{R P}^{2}$ ?

Теория продолжения получила развитие во многих направлениях, она распространена на сепарабельные метрические пространства [7] и даже на более общие пространства [8]. Недавние результаты М. Левина позволяют распространить ее на все метризуемые пространства [9]. Вот некоторые результаты теории продолжения, делающие ее нетривиальной.

Teорема 1 ([4]). Пусть $F: \sigma$-Comp $\rightarrow \sigma$-Comp - нормальный функтор с конечными носителями. Тогда е- $\operatorname{dim} X \leqslant K$ влечет $\mathrm{e}-\operatorname{dim} X \leqslant F(K)$.

ТеОРема 2 ([10]). Для любого сепарабельного метрического пространства $X$ и любого счетного комплекса $K$ c e-dim $X \leqslant K$ существует пополнение $\bar{X}$ пространства $X$ с e-dim $\bar{X} \leqslant K$.

Tеорема $3([5])$. Допустим, что е-dim $X \leqslant K * L^{1}$ для метрического компакта $X$ и счетных комплексов $K$ и L. Тогда существует такое $F_{\sigma}$ множество $Y \subset X$, ито e- $\operatorname{dim} Y \leqslant K u$ e- $\operatorname{dim}(X \backslash Y) \leqslant L$.

\footnotetext{
${ }^{1}$ Здесь $K * L$ обозначает джойн комплексов $K$ и $L$.
} 
Teоpema 4 ([11]). Допустим, что е- $\operatorname{dim} Y \leqslant K u$ e-dim $(X \backslash Y) \leqslant L$ для некоторого подмнохсества $Y \subset X$. Тогда е- $\operatorname{dim} X \leqslant K * L$.

ТеОРема 5 ([12]). Пусть $f: X \rightarrow Y$ нульмерное отображсение компактов, $и$ пусть е-dim $Y \leqslant K$. Тогда е-dim $X \leqslant K$ для любого комплекса $K$.

Все эти результаты, исключая теорему 1, являются аналогами соответствующих результатов в теории размерности. Чтобы помочь читателю увидеть правильную размерно-теоретическую интерпретацию теорем 3 и 4 , заметим, что $S^{k} * S^{l}=S^{k+l+1}$. Теорема 1 применительно к функтору $S P^{\infty}$ бесконечной симметрической степени, играет ключевую роль в доказательстве следующей теоремы.

Теорема 6 ([4]). Для конечномерного компакта $X$ и простого $C W$-комплекса $K$ следующие условия әквивалентны:

(1) $\mathrm{e}-\operatorname{dim} X \leqslant K$,

(2) $\operatorname{dim}_{H_{i}(K)} X \leqslant i$.

Заметим, что в [4] эта теорема сформулирована для односвязного $K$. Однако доказательство, приведенное там, проходит для простого $K$.

Также упомянем два результата из теории продолжения, связанных с метризуемосТЬю.

TеОрема 7 ([13]). Пусть $K$ - некоторый $C W$-комплекс $u X-$ компакт ${ }^{2} c$ е-dim $X \leqslant K$. Допустим, что $X$ является абсолютным әкстензором для класса компактов $Y$ с е- $\operatorname{dim} Y \leqslant K, X \in A E([K])$. Тогда $X$ метризуем.

Teорема 8 ([17]). Пусть $X$ компакт $c$ e-dim $X \leqslant K$ u nусть $f: X \rightarrow Y$ является отображсением в метрическое пространство $Y$. Тогда отображсение $f$ можно факторизовать через метризуемый компакт $Z$ со свойством $\mathrm{e}-\operatorname{dim} Z \leqslant K$.

Оба результата аналогичны соответствующим результатам теории размерности. Теорема 7 является аналогом факта, что всякий $n$-мерньй компактньй абсолютный экстензор в размерности $n$ метризуем [14]. Теорема 8 - аналог факторизационной теоремы Мардешича [2]. В обеих теоремах имеется “естественное" предположение, что $K$ - счетньй комплекс. Недавно Рубин и Шапиро [15], [16] сделали невероятное открытие, что предположение счетности $K$ в этой теореме излишне. В этой работе мы рассматриваем следствия их открытия для теории продолжения.

Пусть $\mathscr{B}$ - класс компактов и пусть $\mathscr{W}$ - класс $C W$-комплексов. Определим предпорядок $\leqslant \mathscr{B}$ на $\mathscr{W}$ так:

$K \leqslant \mathscr{B} L$, если и только если $K \in A E(X) \Longrightarrow L \in A E(X)$ для любого $X \in \mathscr{B}$.

Этот предпорядок определяет отношение эквивалентности $\sim$ на $\mathscr{W}$ и частичньй порядок на $\mathscr{W} / \sim$. Класс комплекса $K$ обозначается $[K]$ и назьвается әкстенсивным типом комплекса $K$. Гомотопически эквивалентные комплексы имеют одинаковые экстенсивные типы (для любого $\mathscr{B}$ ). Обратное неверно: $\left[S^{n}\right]=\left[S^{n} \vee S^{n}\right]$.

\footnotetext{
${ }^{2}$ Компактом в данной статье назьвается компактное хаусдорфово пространство, возможно, неметризуемое.
} 
Заметим, что е- $\operatorname{dim} X \leqslant \bigvee_{\alpha \in A} K_{\alpha} \Longleftrightarrow \mathrm{e}-\operatorname{dim} X \leqslant K_{\alpha}$ для всех $\alpha \in A$. Это влечет, что $\left[\bigvee_{\alpha \in A} K_{\alpha}\right]$ является нижней грањью семейства $\left\{\left[K_{\alpha}\right] \mid \alpha \in A\right\}$. Если $\mathscr{W}$ замкнуто относительно операции взятия букета семейства комплексов, тогда каждое семейство $F \subset \mathscr{W} / \sim$ имеет нижнюю грань. В этом случае мы можем определить экстенсиональную размерность е-dim как функцию на $\mathscr{B}$, принимающую значения в $\mathscr{W} / \sim$, так что e- $\operatorname{dim} X=\inf \{[K] \mid$ e-dim $X \leqslant K\}$. Тогда свойство е-dim $X \leqslant K$ в точности означает неравенство е-dim $X \leqslant \mathscr{B}[K]$.

Для класса $\mathscr{W}^{s}$ односвязных (или, более общо, простых) $C W$-комплексов и конечномерных компактов $\mathscr{F}$ упорядоченное множество $\left(\mathscr{W}^{s}, \leqslant \mathscr{F}\right)$ хорошо изучено ([6], [17]-[19]). В этой статье мы рассматриваем класс $\mathscr{W}$ всех $C W$-комплексов и класс $\mathscr{C}$ всех компактов. Через $\mathscr{W}_{0}$ и $\mathscr{C}_{0}$ обозначаем класс всех счетных $C W$-комплексов и класс метризуемых компактов соответственно. Известно, что отображение e-dim: $\mathscr{C} \rightarrow \mathscr{W} / \sim$ эпиморфно. Следуюший вопрос до сих пор открыт.

Провлема 2 ([20]). Можно ли е- $\operatorname{dim} X$ реализовать посредством счетного комплекса для метрического компакта $X$ ?

В теории продолжения имеется еще не формализованная двойственность между компактами и $C W$-комплексами [20]. Двойственной к проблеме 2 является

ПроБлема 3. Дан счетный комплекс К. Существует ли метризуемый комnaкm $X \quad c$ e-dim $X=[K]$ ?

\section{§. Теорема Рубина-Шапиро и двойственная к ней}

В этом параграфе мы дадим доказательство факторизационной теоремы РубинаШапиро, которое можно естественным образом дуализовать, и затем докажем двойственную теорему.

Teopema 9 ([15], [16]). Допустим, что $M \in \mathscr{W}, X \in \mathscr{C} u$ e- $\operatorname{dim} X \leqslant M$. Тогда для любого отображения $f: X \rightarrow Y$ c $Y \in \mathscr{C}_{0}$ найдется $Z \in \mathscr{C}_{0} c \mathrm{e}-\operatorname{dim} Z \leqslant M u$ факторизация $f=q \circ g$, где $g: X \rightarrow Z u q: Z \rightarrow Y$.

Теорема 8 отличается от теоремы 9 предположением $M \in \mathscr{W}$. Двойственная теорема утверждает следуюшее.

Teоpema 10. Допустим, ито $M \in \mathscr{W}, X \in \mathscr{C} u \mathrm{e}-\operatorname{dim} X \leqslant M$. Тогда для любого подкомплекса $L \subset M c L \in \mathscr{W}_{0}$ найдется подкомплекс $K \in \mathscr{W}_{0} c \mathrm{e}-\operatorname{dim} X \leqslant$ $K$ u $L \subset K \subset M$.

Последовательность $C \stackrel{\phi}{\longrightarrow} L \stackrel{j}{\longrightarrow} K$ отображений, где $C \in \mathscr{C}_{0}$ и $j: L \rightarrow K$ является включением подкомплекса в конечный $C W$-комплекс $K$, мы называем строкой. Две такие строки $C \stackrel{\phi}{\longrightarrow} L \stackrel{j}{\rightarrow} K$ и $C \stackrel{\phi^{\prime}}{\longrightarrow} L^{\prime} \stackrel{j}{\longrightarrow} K^{\prime}$ назьваются әквивалентнылми, если существует гомотопическая эквивалентность пар $h:(K, L) \rightarrow\left(K^{\prime}, L^{\prime}\right)$, производящая из них гомотопически коммутативную диаграмму.

ПрЕДЛОЖЕНИЕ 1. Для любого $C \in \mathscr{C}_{0}$ имеется только счетное число неэквивалентных строк $C \stackrel{\phi}{\longrightarrow} L \stackrel{j}{\rightarrow} K$. 
ДокАЗАТЕЛЬСТвО. Множество гомотопических классов $[C, L]$ счетно для любого метрического компакта $C$ и конечного комплекса $L$. Число конечных гомотопически различных пар конечных симплициальных комплексов $(K, L)$ счетно. Так как каждая конечная $C W$-пара гомотопически эквивалентна паре конечных симплициальных комплексов, число различных строк счетно.

Зафиксируем счетную базу топологии $\left\{C_{k}\right\}=\mathscr{B}$ на гильбертовом кубе $Q$, состояшую из замкнутых множеств. Дополнительно предположим, что семейство $\mathscr{B}$ замкнуто относительно конечных объединений.

ДокАЗАТЕЛЬСтво теОРемы 9. Рассмотрим вложение $Y \subset Q$ и занумеруем все классы строк $C \stackrel{\phi}{\longrightarrow} L \stackrel{j}{\rightarrow} K, C \in \mathscr{B}$, со следуюшим свойством:

$(* *)$ существует продолжсение $\psi: X \rightarrow K$ отображения $\left.j \circ \phi \circ f\right|_{f^{-1}(Y \cap C)}$.

Заметим, что свойство $(* *)$ инвариантно относительно отношения эквивалентности. Выберем по одному представителю $C \stackrel{\phi}{\longrightarrow} L \stackrel{j}{\longrightarrow} K$ из каждого класса строк и зафиксируем семейство соответствуюших продолжений $\left\{\psi_{i}: X \rightarrow K_{i}\right\}$. Так как это семейство счетно, согласно факторизационной теореме имеется метрический компакт $Z_{1}$ с отображением $f_{1}: X \rightarrow Z_{1}, f_{0}^{\prime}: Z_{1} \rightarrow Y$ и семейство $\left\{\psi_{i}^{\prime}: Z_{1} \rightarrow K_{i}\right\}$ такое, что $f=f_{0}^{\prime} \circ f_{1}$ и $\psi_{i}=\psi_{i}^{\prime} \circ f_{1}$, т.е. $\psi_{i}^{\prime}$ является продолжением отображения $\left.j \circ \phi \circ f_{0}^{\prime}\right|_{\left(f_{0}^{\prime}\right)^{-1}(Y \cap C)}$. Теперь рассматриваем вложение $Z_{1} \subset Q$ и повторяем процедуpy.

В результате мы построим обратную последовательность компактов $\left\{Z_{i} ; f_{i}^{i+1}\right\}_{i \geqslant 0}$ с $Z_{0}=Y$ и $Z=\lim _{\leftarrow}\left\{Z_{i} ; f_{i}^{i+1}\right\}$ такую, что отображение $f$ факторизуется через $Z$, т.е. имеется отображение $g: X \rightarrow Z$ с $f_{0}^{\infty} \circ g=f$. По построению, пространство $Z$ имеет следующее свойство:

(***) для всякого $і$ и в всякой строки $C \stackrel{\phi}{\longrightarrow} L \stackrel{j}{\rightarrow} K$ со свойством $(* *)$ для $Z_{i}$ существует продолжение $\bar{\psi}: Z \rightarrow K$ отображения $\left.j \circ \phi \circ f_{i}^{\infty}\right|_{\left(f_{i}^{\infty}\right)^{-1}\left(Z_{i} \cap C\right)}$.

Положим $q=f_{0}^{\infty}$ и проверим условие е- $\operatorname{dim} Z \leqslant M$. Пусть $\alpha: A \rightarrow M$ - задача продолжения. В силу компактности $A$, найдется конечный подкомплекс $L \subset M$ с $\phi(A) \subset L$. Так как $L \in A N E$, для достаточно большого $i$ можно найти $C \in \mathscr{B}$ и отображение $\alpha_{i}: C \rightarrow L$ так, что $\left(f_{i}^{\infty}\right)^{-1}(C) \supset A$ и композиция $\left.\alpha_{i} \circ f_{i}^{\infty}\right|_{A}$ гомотопна $\alpha$. В силу того, что е- $\operatorname{dim} X \leqslant M$, существует продолжение $\psi: X \rightarrow M$ отображения $\left.\alpha_{i} \circ f_{i}^{\infty} \circ g\right|_{g^{-1}\left(f_{i}^{\infty}\right)^{-1}\left(Z_{i} \cap C\right)}$. Так как $X-$ компакт, существует конечный подкомплекс $K \subset M$, содержаший $\psi(X)$. Итак, строка $C \stackrel{\alpha_{i}}{\rightarrow} L \stackrel{j}{\rightarrow} K$ имеет свойство $(* *)$. В силу (***) имеется продолжение $\bar{\psi}: Z \rightarrow K$ отображения $\left.j \circ \alpha_{i} \circ f_{i}^{\infty}\right|_{\left(f_{i}^{\infty}\right)^{-1}\left(Z_{i} \cap C\right)}$. Следовательно, $\bar{\psi}$ является продолжением отображения $\left.j \circ \alpha_{i} \circ f_{i}^{\infty}\right|_{A}$. Теорема о продолжении гомотопии влечет продолжаемость $\alpha$.

ДокАЗАТЕЛЬСТво ТЕОРЕмЫ 10. Пусть класс $\mathscr{B}$ - такой же, как и вьше, и пусть $C, C^{\prime} \in \mathscr{B}$. Определим дуальную строку как диаграмму $C^{\prime} \stackrel{j}{\longleftarrow} C \stackrel{\phi}{\longrightarrow} N$, где $j$-вложение и $N$ - конечный $C W$-комплекс. Мы называем две дуальные строки $C^{\prime} \stackrel{j}{\longleftarrow} C \stackrel{\phi}{\longrightarrow} N$ 
и $C^{\prime} \stackrel{j}{\longleftarrow} C \stackrel{\phi^{\prime}}{\longrightarrow} N^{\prime}$ эквивалентными, если существует гомотопическая эквивалентность $h: N \rightarrow N^{\prime}$, производящая из этих строк гомотопически коммутативную диаграмму. Сушествует только счетное число различных дуальных строк. Мы перенумеруем классы эквивалентности дуальных строк со следуюшим свойством:

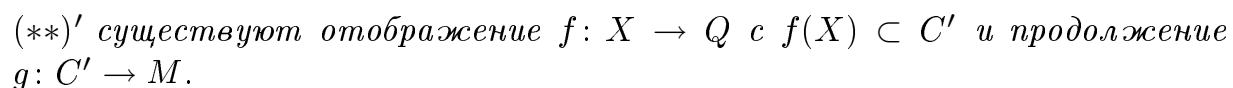

Это свойство при наших условиях инвариантно. Для любого из перенумерованных классов зафиксируем продолжение $g$ для некоторой дуальной строки $C^{\prime} \stackrel{j}{\longleftarrow} C \stackrel{\phi}{\longrightarrow} N$, представляющей этот класс и конечный подкомплекс $K_{j, \phi} \subset M$ с $K_{j, \phi} \supset g\left(C^{\prime}\right)$. Полагаем $K_{0}=L, K_{1}=K_{0} \cup K_{j_{1}, \phi_{1}}$, где $K_{j_{1}, \phi_{1}}$ из первого класса и т. д. В результате получится счетное объединение счетных подкомплексов $L=K_{0} \subset K_{1} \subset K_{2} \subset \cdots$. Положим $K=\bigcup_{i=0}^{\infty} K_{i}$. Покажем, что е- $\operatorname{dim} X \leqslant K$. Пусть $\alpha: A \rightarrow K$ - задача продолжения на $X$, тогда $\alpha(A)$ лежит в конечном подкомплексе $N$. Рассмотрим продолжение $\bar{\alpha}: X \rightarrow M$. Так как образ $\bar{\alpha}(X)$ лежит в конечном подкомплексе $M^{\prime}$, отображение $\bar{\alpha}$ может быть пропущено через метрический компакт $Y$ так что $\bar{\alpha}=g^{\prime} \circ f$, $f: X \rightarrow Y$. Рассмотрим вложение $Y \subset Q$ и продолжение $g: C^{\prime} \rightarrow M^{\prime}$ отображения $g^{\prime}: Y \rightarrow M^{\prime}, C^{\prime} \in \mathscr{B}$. Мы можем допустить, что $N \subset M^{\prime}$. Пусть $r: R \rightarrow N-$ деформационная ретракция замкнутой регулярной окрестности подкомплекса $N$ в $M^{\prime}$. Возьмем $C \in \mathscr{B}$ такое, что $f(A) \subset C \subset g^{-1}(\operatorname{Int}(R))$. Заметим, что дуальная строка $C^{\prime} \stackrel{j}{\longleftarrow} C \stackrel{\phi}{\longrightarrow} N$ удовлетворяет условию $(* *)^{\prime}$, где $\phi=\left.r \circ g\right|_{C}$. Найдется занумерованная дуальная строка $C^{\prime} \stackrel{j}{\longleftarrow} C \stackrel{\phi^{\prime}}{\longrightarrow} N^{\prime}$, эквивалентная строке $C^{\prime} \stackrel{j}{\longleftarrow} C \stackrel{\phi}{\longrightarrow} N$, использованной при построении $K$. Пусть $\bar{g}: C^{\prime} \rightarrow M$ обозначает продолжение отображения $\phi^{\prime}$. По построению $K$, имеем $\bar{g}\left(C^{\prime}\right) \subset K$. Тогда $\phi$ продолжается на $K$. Заметим, что отображение $\left.\phi\right|_{f(A)}$ гомотопно $\left.g\right|_{f(A)}=\left.g^{\prime}\right|_{f(A)}$ как отображение в $K$. Следовательно, $\left.g^{\prime}\right|_{f(A)}$ имеет продолжение, откуда $\alpha=\left.g^{\prime} \circ f\right|_{A}$ продолжаемо.

\section{$\S$ 3. Теорема о букете}

TeOpema $11 . K \leqslant_{\mathscr{C}} M \Longleftrightarrow K \leqslant_{\mathscr{C}_{0}} M$ для $K, M \in \mathscr{W}$.

ДоказАТЕЛЬство. Ясно, что $K \leqslant \mathscr{C} M$ влечет $K \leqslant \mathscr{C}_{0} M$. Допустим, что верно $K \leqslant \mathscr{C}_{0} M$, и покажем, что $K \leqslant{ }_{\mathscr{C}} M$. Пусть $X-$ неметризуемый компакт с е-dim $X \leqslant K$. Используя теорему 9 , мы можем представить $X$ как предельное пространство обратного сигма-спектра $X=\lim _{\leftarrow}\left\{X_{\alpha}\right\}$ [21] метрических компактов с е- $\operatorname{dim} X_{\alpha} \leqslant K$. Согласно предположениям, отсюда следует, что е- $\operatorname{dim} X_{\alpha} \leqslant M$. Теорема об обратном пределе (см., например, [20]) влечет, что е- $\operatorname{dim} X \leqslant M$.

Двойственной к теореме 11 является следуюшая

ТеОрема 12. Неравенство е- $\operatorname{dim} X \leqslant \mathrm{e}-\operatorname{dim} Y$ справедливо, если и только если е-dim $Y \leqslant L$ влечет е- $\operatorname{dim} X \leqslant L$ для любого счетного комплекса $L$. 
ДокАЗАТЕльствО. В направлении $(\Rightarrow)$ это очевидно. Для доказательства теоремы в другом направлении достаточно показать, что е- $\operatorname{dim} Y \leqslant M$ влечет е- $\operatorname{dim} X \leqslant$ $M$ для любого комплекса $M$. В силу теоремы 10 , неравенство е- $\operatorname{dim} Y \leqslant M$ влечет представимость $M$ в виде прямого предела $M=\lim _{\rightarrow} M_{\alpha}$ счетных комплексов c е-dim $Y \leqslant M_{\alpha}$. Тогда по предположению имеем е- $\operatorname{dim} X \leqslant M_{\alpha}$. В силу теоремы о прямом пределе (двойственной теореме об обратном) [20] можно заключить, что e- $\operatorname{dim} X \leqslant M$.

Следуюшая теорема является главньм результатом этой статьи.

Теорема 13. Каждый $C W$-комплекс $K$ әкстенсионально әквивалентен букету счетных комплексов $[K]=\left[\bigvee_{\alpha \in A} L_{\alpha}\right]$, где мощность $A$ не превосходит континуума.

ДокаЗАТЕЛЬСтво. Положим $A=\left\{L \in \mathscr{W}_{0} \mid K \leqslant L\right\}$. Покажем, что $[K]=$ $\left[\bigvee_{L \in A} L\right]$. Так как неравенство е- $\operatorname{dim} X \leqslant \bigvee_{L \in A} L$ равносильно системе неравенств е- $\operatorname{dim} X \leqslant L, L \in A$, отсюда следует, что $K \leqslant \bigvee_{L \in A} L$. Допустим, что $K<\bigvee_{L \in A} L$. Так как е-dim эпиморфно [17], найдутся компакты $X$ и $Y$ с е- $\operatorname{dim} X=\bigvee_{L \in A} L$ и e-dim $Y=K$. Так как неравенство е- $\operatorname{dim} X \leqslant \mathrm{e}-\operatorname{dim} Y$ не выполнено, теорема 12 влечет существование счетного комплекса $L$ такого, что $K=\mathrm{e}-\operatorname{dim} Y \leqslant L$ и е- $\operatorname{dim} X \nless L$. Неравенство $K \leqslant L$ влечет, что $L \in A$. Следовательно, е- $\operatorname{dim} X \leqslant L$. Противоречие.

\section{СПИСОК ЛИТЕРАТУРЫ}

[1] Spanier E. Algebraic Topology. New York: McGraw-Hill, 1966.

[2] Engelking R. Dimension Theory. Amsterdam: North-Holland, 1978.

[3] Dranishnikov A. N. On intersection of compacta in Euclidean space II // Proc. Amer. Math. Soc. 1991. V. 113. P. 1149-1154.

[4] Dranishnikov A. N. Extension of maps into CW complexes // Math. USSR Sbornik. 1993. V. 74. P. 47-56.

[5] Dranishnikov A.N. On the mapping intersection problem // Pacific J. Math. 1996. V. 173. №2. P. 403-412.

[6] Dranishnikov A. N. On the dimensions of the product, the union and the intersection of two spaces // Preprint, 1995.

[7] Dranishnikov A. N., Dydak J. Extension theory of separable metric spaces with applications to dimension theory // Preprint, 1995.

[8] Chigogidze A. Infinite dimensional topology and shape theory // Preprint, 1997.

[9] Levin M. On extensional dimension of metrizable spaces // Preprint, 1998.

[10] Olszewski W. Completion theorem for cohomological dimensions // Proc. Amer. Math. Soc. 1995. V. 123. P. 2261-2264.

[11] Dydak J. Cohomological dimension and metrizable spaces II // Trans. Amer. Math. Soc. 1996. V. 348. P. $1647-1661$.

[12] Dranishnikov A.N., Uspenskij V.V. Light maps and extensional dimension // Topology Appl. 1997. V. 80. № 1-2. P. 91-100.

[13] Chigogidze A., Zarichnyi M. On absolute extensors modulo a complex // Topology Appl. (to appear).

[14] Дранишников А. Н. Абсолютные экстензоры в размерности $n$ и $n$-мерные отображения, повышающие размерность // УМН. 1984. Т. 39. № 5. С. 55-95.

[15] Rubin L., Shapiro Ph. The Mardešić factorization theorem for extension theory // Preprint, 1998. 
[16] Levin M., Rubin L., Shapiro Ph. The Mardešić factorization theorem for extension theory and $C$-separation // Preprint, 1998.

[17] Dranishnikov A. N. Eilenberg-Borsuk theorem for maps into arbitrary complexes // Math. Sbornik. 1994. V. 185. P. 81-90.

[18] Dranishnikov A. N. Basic elements of the cohomological dimension theory of compact metric spaces // Preprint, 1997.

[19] Dranishnikov A. N., Repovš D., Schepin E. V. Transversal intersection formula for compacta // Preprint, 1996.

[20] Dranishnikov A.N., Dydak J. Extension dimension and extension type // Proc. Steklov Institute of Math. 1996. V. 212. P. 55-88.

[21] Шепин Е. В. Топология пределшных пространств несчетных обратных спектров // УМН. 1976. V. 31. № 5. P. 191-226.

Университет Флориды,

Математический институт им. В. А. Стеклова РАН

Поступила в редакцию

E-mail: dranish@math.ufl.edu 